\title{
CARACTERÍSTICAS DO CARVÃO DE UM CLONE DE Eucalyptus grandis W. Hill ex Maiden x Eucalyptus urophylla S. T. Blake
}

\author{
Marina Donária Chaves Arantes ${ }^{1 *}$, Paulo Fernando Trugilho², \\ José Reinaldo Moreira da Silva², Carlos Rogério Andrade ${ }^{3}$
}

*Autora para correspondência: mdonariac@hotmail.com

\begin{abstract}
RESUMO: Neste trabalho, objetivou-se determinar a variabilidade existente nas características do carvão de um clone de Eucalyptus grandis x Eucalyptus urophylla aos 6 anos, plantado no município de Martinho Campos, MG. As árvores foram distribuídas em três classes de diâmetro e a amostragem consistiu na retirada de discos, de $2,5 \mathrm{~cm}$ de espessura, a $2 \%, 10 \%, 30 \%$ e $70 \%$ da altura comercial, além de um a $1,30 \mathrm{~m}$ do solo (DAP) e, a partir deste ponto, de metro em metro até a altura comercial. Foram tomadas amostras ao longo do raio nos discos, considerando os dois lados em relação à medula. As amostras foram carbonizadas em forno elétrico de laboratório, sendo determinados os rendimentos e a qualidade do carvão vegetal produzido. Pelos resultados, verificouse que para a primeira carbonização, a classe diamétrica influenciou significativamente o rendimento gravimétrico em carvão vegetal, em líquido pirolenhoso e o teor de cinza do carvão vegetal produzido, e que o rendimento em gás não condensável, o teor de materiais voláteis e o de carbono fixo não foram influenciados pela classe diamétrica; para a segunda carbonização o rendimento gravimétrico aumentou da casca para a medula, para todas as classes diamétricas e ocorreu redução dessa característica com a diminuição das classes diamétricas e que os valores de densidade relativa aparente tenderam a aumentar no sentido medula-casca, em todas as classes diamétricas.
\end{abstract}

Palavras-chave: Carvão vegetal, densidade relativa aparente, classe diamétrica.

\section{CHARCOAL CHARACTERISTICS OF AN Eucalyptus grandis W. Hill ex Maiden x Eucalyptus urophylla S. T. Blake CLONE}

ABSTRACT: This work determined the variability existent in the characteristics of the charcoal of a clone of Eucalyptus grandis $x$ Eucalyptus urophylla at 6 years of age, planted in the municipal district of Martinho Campos, MG. The trees were distributed into three diameter classes and the sampling consisted of the removal of 2,5 cm thick disks at 2\%, 10\%, 30\% and $70 \%$ of the commercial height, besides one at $1.30 \mathrm{~m}$ from the soil $(D B H)$ and starting from this point meter by meter until the commercial height. Samples were taken along the disk radius, considering the two sides in relation to the core. The samples were carbonized in an electric laboratory oven and the yield and the quality of the produced charcoal were determined. It was concluded that for the first carbonization, the diametric class significantly influenced the gravimetric yield in wood charcoal and in pyroligneous liquid, the ash level of the charcoal produced. The non-condensable gas yield, the level of volatile materials and that of fixed carbon were not influenced by the diametric class. For the second carbonization, the gravimetric yield of charcoal increased from the bark to the pith, in all diametric classes and there was a reduction for this characteristic with the decreasing diametric classes and the apparent relative density values tended to increase from pith to bark in all diameter classes.

Key words: Charcoal, eucalyptus, diametric class.

\section{INTRODUÇÃO}

O processo de carbonização pode ser definido como a decomposição térmica da madeira na ausência ou na presença controlada de ar ou oxigênio, resultando em duas fases: uma sólida, que é o carvão vegetal e uma gasosa, composta por gases condensáveis e gases não condensáveis.

Industrialmente, o carvão vegetal é o mais importante combustível e redutor do minério de ferro, em operações siderúrgicas e metalúrgicas. Os primitivos processos de metalurgia de ferro se iniciaram apoiados

${ }^{1}$ Universidade Federal do Espírito Santo - Alegre, Espírito Santo, Brasil

${ }^{2}$ Universidade Federal de Lavras - Lavras, Minas Gerais, Brasil

${ }^{3}$ Universidade Federal de Goiás - Jataí, Goiás, Brasil no carvão vegetal, quando ainda nem se pensava na utilização do carvão mineral para a obtenção do coque em operações industriais. A utilização do carvão vegetal, no Brasil, apresenta inúmeras vantagens em relação ao carvão mineral: é renovável, menos poluente, tem baixo teor de cinza, é praticamente isento de enxofre e fósforo, é mais reativo, o processo de produção e transporte não é centralizado e propicia a poupança de divisas com a eliminação de importações de combustíveis fósseis. O carvão vegetal também tem sido utilizado nas indústrias de cimento, cal e cerâmicas (EUCALIPTO..., 2003).

Cerne, Lavras, v. 19, n. 3, p. 423-431, jul./set. 2013 
Para a produção do carvão, a madeira sofre uma degradação térmica que reflete a soma das respostas térmicas de seus três principais componentes: hemiceluloses, celulose e lignina. Destes, as hemiceluloses e a celulose representam cerca de $70 \%$ da composição da madeira e são os componentes menos estáveis e se degradam entre $225^{\circ}$ e $375^{\circ} \mathrm{C}$. Pode-se deduzir que a perda acentuada de massa deve-se à degradação desses dois componentes. A lignina é mais estável e se degrada de modo mais lento, sendo a principal responsável pela formação do carvão vegetal (GOLDSTEIN, 1977). Segundo o mesmo autor, para temperaturas inferiores a $375^{\circ} \mathrm{C}$, o rendimento em gases condensáveis varia em função da temperatura, em razão da estabilidade térmica dos componentes da madeira que na decomposição irão constituir os gases condensáveis e que, acima de $450^{\circ} \mathrm{C}$, a formação de gases condensáveis é desprezível, pois nessa temperatura a lignina atinge seu pico máximo de decomposição.

Pinheiro et al. (2005) estudaram a influência da temperatura e da taxa de aquecimento da carbonização nas propriedades do carvão vegetal de Eucalyptus camaldulensis, E. cloeziana e Corimbia citriodora, com idades entre 6,5 e 7,5 anos e verificaram que, para todas as espécies analisadas, quanto menor a temperatura e a taxa de aquecimento, maior é o rendimento da carbonização. Os mesmos autores concluíram que a temperatura ótima do processo de carbonização foi na faixa de $300^{\circ}-450^{\circ} \mathrm{C}$, para todas as espécies analisadas, tanto do ponto de vista quantitativo - rendimento gravimétrico, quanto do ponto de vista qualitativo - teor de carbono fixo.

Para qualificar e predizer uma melhor utilização do carvão vegetal é necessário que este seja avaliado. Para isso, existem procedimentos e normas a serem seguidas. Um exemplo é a análise química imediata do carvão vegetal, na qual se determinam a umidade, os teores de materiais voláteis, carbono fixo e cinza.

Outra característica muito importante para avaliar a qualidade do carvão é a densidade que, segundo Mendes et al. (1982), afetará as suas demais propriedades e, ainda, que a densidade do carvão é variável em função de suas características, como granulometria e presença de trincas. Fatores relacionados com o processo de carbonização podem afetar a densidade do carvão produzido, como a temperatura final de carbonização e a velocidade de aquecimento.

A amostragem realizada na madeira para a produção de carvão, pode influenciar na qualidade deste. Nesse sentido, Trugilho et al. (2005) avaliaram os rendimentos e as características do carvão vegetal em função da posição radial de amostragem na madeira de seis clones de Eucalyptus spp. Esses autores observaram tendência de maior produção de carvão vegetal nas posições intermediária e central, exceto para dois clones e também que a densidade relativa aparente do carvão apresentou comportamento inverso, ou seja, foi maior na posição radial externa e menor na posição radial central, exceto para um clone. Esse comportamento era esperado, uma vez que a densidade da madeira apresenta tendência de aumento do centro para a periferia do tronco.

Diante do exposto, neste trabalho, objetivou-se determinar a variabilidade existente nas características do carvão de um clone de Eucalyptus grandis x Eucalyptus urophylla aos 6 anos.

\section{MATERIAL E MÉTODOS}

\subsection{Primeira carbonização - amostras retiradas de metro em metro}

Para a carbonização em escala de laboratório foi utilizado o material proveniente da amostragem de metro em metro. De cada uma das cinqüenta árvores, em todas as classes diamétricas $(14,2 ; 11,4$ e $8,1 \mathrm{~cm})$, foram retirados discos de 2,5 cm de espessura nas posições da base, $1,3 \mathrm{~m}$ de altura do solo (DAP) e, a partir desse ponto, de metro em metro, até a altura comercial do tronco, considerado até $5 \mathrm{~cm}$ de diâmetro. Dos discos foram utilizadas duas cunhas opostas para a carbonização.

As carbonizações foram realizadas em um forno elétrico (mufla) adaptado (Figura 1). O controle do aquecimento foi manual, com incrementos de $50^{\circ} \mathrm{C}$ a cada 30 minutos, o que corresponde a uma taxa média de $1,67^{\circ} \mathrm{C}$ por minuto. A temperatura inicial foi sempre igual a $150^{\circ} \mathrm{C}$ e a temperatura máxima, de $450^{\circ} \mathrm{C}$, permanecendo estabilizada por um período de 30 minutos. O tempo total da carbonização foi, portanto, de 4 horas.

Após cada carbonização, foi determinado o rendimento gravimétrico em carvão, líquido pirolenhoso e, por diferença, gases não-condensáveis, todos em relação à massa da madeira seca. Foi determinado, também, o rendimento em carbono fixo.

Realizou-se a análise química imediata do carvão para determinar o teor de materiais voláteis, cinza e de carbono fixo, conforme a Norma NBR 8112 (ASSOCIAÇÃO BRASILEIRA DE NORMAS TÉCNICAS - ABNT, 1983).

Em razão da pequena quantidade de carvão produzida, a densidade relativa aparente do carvão vegetal foi determinada pelo método hidrostático, por meio da imersão em água. A densidade relativa aparente média foi

Cerne, Lavras, v. 19, n. 3, p. 423-431, jul./set. 2013 


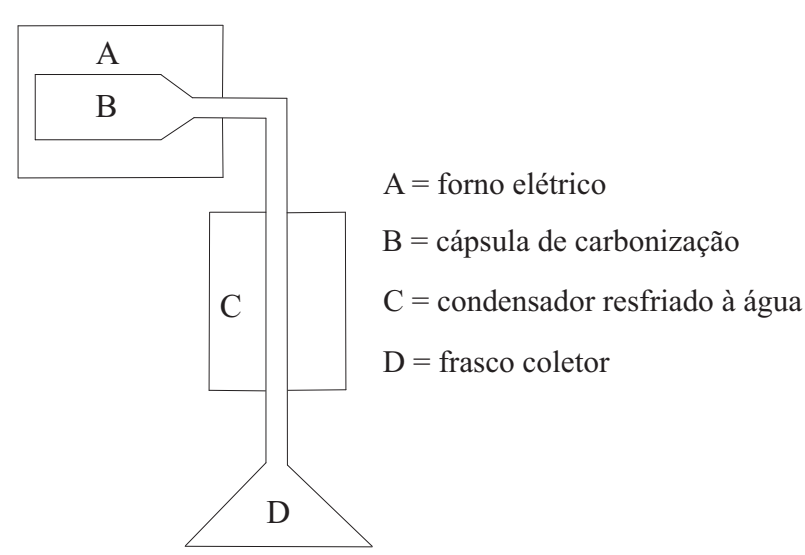

Figura 1 - Esquema do equipamento utilizado nas carbonizações.

Figure 1 - Scheme of the equipment used in the carbonization.

considerada como sendo a média aritmética, levando-se em consideração todos os pontos de amostragem longitudinal ao longo do tronco, para cada árvore.

\subsection{Segunda carbonização - amostras retiradas no DAP e posições longitudinais}

De cada uma das cinqüenta árvores, em todas as classes diamétricas $(14,2 ; 11,4$ e $8,1 \mathrm{~cm})$, foram retirados discos de 2,5 cm de espessura nas posições a $1,30 \mathrm{~m}$ de altura do solo (DAP) e a $2 \%, 10 \%, 30 \%$ e $70 \%$ da altura comercial, considerada até $5 \mathrm{~cm}$ de diâmetro, sendo amostrados os dois lados em relação à medula, em que o número de amostras radiais retiradas foi dependente do diâmetro do disco. Para facilitar a operação de carbonização das amostras, as mesmas foram agrupadas em feixes, como pode ser observado na Figura 2.

As carbonizações foram realizadas em um forno elétrico (mufla) adaptado. O controle do aquecimento foi digital, com incrementos de $15^{\circ} \mathrm{C}$ a cada 30 minutos, o que corresponde a uma taxa média de $0,5^{\circ} \mathrm{C}$ por minuto. A temperatura inicial foi sempre igual a $150^{\circ} \mathrm{C}$ e a temperatura máxima de $450^{\circ} \mathrm{C}$, permanecendo estabilizada por um período de 30 minutos. O tempo total de carbonização foi, portanto, de 10 horas.

Após cada carbonização, para as amostras retiradas no DAP, foram determinados os rendimentos gravimétricos e a densidade relativa aparente, sendo esta última característica determinada também para as amostras retiradas nas posições de $2 \%, 10 \%, 30 \%$ e $70 \%$ da altura comercial, em três árvores, sendo uma em cada classe diamétrica. Foi utilizado o procedimento de imersão em água, descrito na norma NBR 11941 (ABNT, 2003).

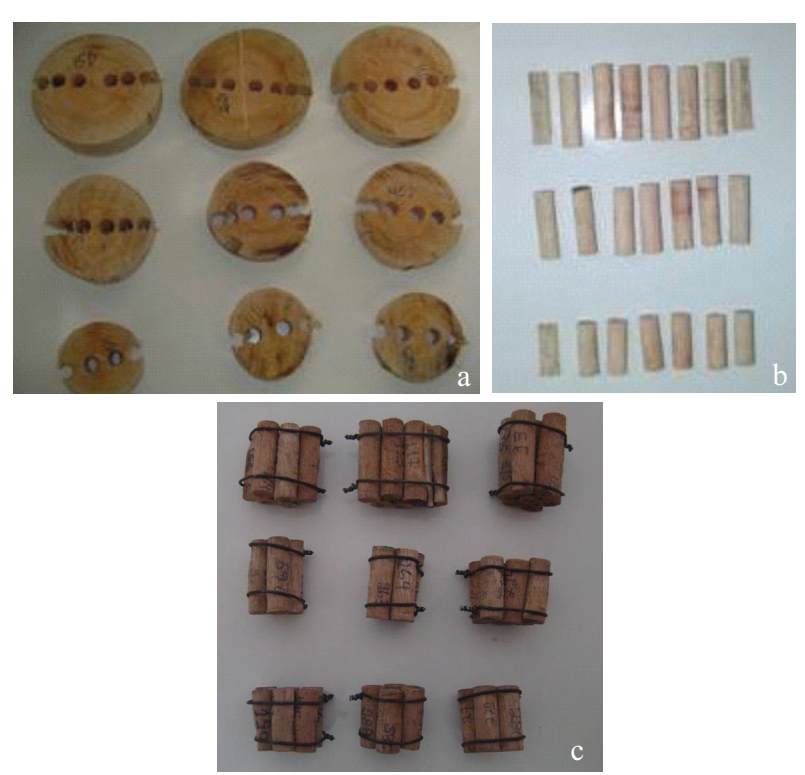

Figura 2 - Esquema da retiradas das amostras radiais nos discos (a), amostras (b) e feixes para carbonização (c).

Figure 2 -Scheme of the radial sample removal from the disks (a), samples (b) and bundles for carbonization (c).

\section{RESULTADOS E DISCUSSÃO}

\subsection{Primeira carbonização - características quantitativas do carvão vegetal}

Os valores médios das características quantitativas da carbonização da madeira encontram-se na Tabela 1 .

Tabela 1 - Valores médios para as características quantitativas da carbonização da madeira do clone de Eucalyptus grandis W. Hill ex Maiden x Eucalyptus urophylla S. T. Blake.

Table 1 - Average values for the quantitative characteristics of the carbonization of wood of the Eucalyptus grandis W. Hill ex Maiden x Eucalyptus urophylla S. T. Blake clone.

\begin{tabular}{cccc}
\hline \multirow{2}{*}{$\begin{array}{c}\text { Classe } \\
(\mathrm{cm})\end{array}$} & \multicolumn{3}{c}{ Rendimento gravimétrico (\%) } \\
\cline { 2 - 4 } & Carvão & Líquido pirolenhoso & Gases* \\
\hline \multirow{2}{*}{8,1} & 33,68 & 48,27 & 18,05 \\
& $(3,30)$ & $(6,98)$ & $(15,26)$ \\
\hline \multirow{2}{*}{11,4} & 34,35 & 48,32 & 17,33 \\
& $(2,94)$ & $(4,12)$ & $(10,32)$ \\
\hline \multirow{2}{*}{14,2} & 35,07 & 46,69 & 18,24 \\
& $(3,11)$ & $(3,81)$ & $(12,84)$ \\
\hline
\end{tabular}

Valores entre parênteses representam os coeficientes de variação (\%). * Gases não condensáveis.

Cerne, Lavras, v. 19, n. 3, p. 423-431, jul./set. 2013 
Observa-se que o rendimento gravimétrico em carvão vegetal apresentou tendência de aumento com a classe diamétrica, enquanto os rendimentos em líquido pirolenhoso e gases não condensáveis não apresentaram tendência definida. Verifica-se que os coeficientes de variação podem ser considerados baixos para o rendimento gravimétrico em carvão e em líquido pirolenhoso, sendo mais elevados para os gases não condensáveis. $\mathrm{O}$ menor rendimento, observado nas classes de menor diâmetro, pode estar relacionado com o processo de cernificação da madeira. Nas classes de menor diâmetro, a porcentagem de cerne é menor que a de alburno, o que pode estar relacionado ao menor rendimento em carvão. Trugilho et al. (2005) encontraram maiores rendimentos em carvão na madeira obtida na porção central do lenho.

\subsubsection{Avaliação física e química imediata do carvão vegetal}

Na Tabela 2, estão apresentados os valores médios de densidade relativa aparente e da análise química imediata do carvão vegetal. Observa-se que o efeito de classe diamétrica somente foi significativo para o teor de cinza no carvão vegetal. Verifica-se, também, a baixa magnitude dos coeficientes de variação, exceto para a umidade e o teor de cinza. A maior variação observada no teor de umidade ocorreu, pelo fato de o carvão ficar guardado, esperando para análise, em recipiente não lacrado, o que provocou o seu umedecimento com o passar do tempo.

Tabela 2 - Valores médios da densidade relativa aparente (DRA) e análise química imediata do carvão do clone de Eucalyptus grandis W. Hill ex Maiden x Eucalyptus urophylla S. T. Blake.

Table 2 - Average apparent relative density values (ARD) and immediate chemical analysis of the charcoal of the Eucalyptus grandis W. Hill ex Maiden x Eucalyptus urophylla S. T. Blake clone.

\begin{tabular}{|c|c|c|c|c|c|}
\hline \multirow{2}{*}{$\begin{array}{l}\text { Classe } \\
(\mathrm{cm})\end{array}$} & \multirow{2}{*}{$\begin{array}{c}\text { DRA } \\
\left(\mathrm{g} / \mathrm{cm}^{3}\right)\end{array}$} & \multicolumn{4}{|c|}{ Teor $(\%)$} \\
\hline & & Umidade & $\begin{array}{l}\text { Materiais } \\
\text { voláteis }\end{array}$ & Cinza & $\begin{array}{c}\text { Carbono } \\
\text { fixo }\end{array}$ \\
\hline 8,1 & $\begin{array}{c}0,368 \mathrm{~A} \\
(4,53)\end{array}$ & $\begin{array}{c}4,09 \\
(36,34)\end{array}$ & $\begin{array}{c}26,72 \mathrm{~A} \\
(8,60)\end{array}$ & $\begin{array}{c}0,21 \mathrm{~A} \\
(36,79)\end{array}$ & $\begin{array}{c}73,07 \mathrm{~A} \\
(3,21)\end{array}$ \\
\hline 11,4 & $\begin{array}{c}0,361 \mathrm{~A} \\
(6,12)\end{array}$ & $\begin{array}{c}3,54 \\
(27,43)\end{array}$ & $\begin{array}{c}26,99 \mathrm{~A} \\
(8,98)\end{array}$ & $\begin{array}{c}0,17 \text { AB } \\
(21,95)\end{array}$ & $\begin{array}{c}72,84 \mathrm{~A} \\
(3,34)\end{array}$ \\
\hline 14,2 & $\begin{array}{c}0,364 \mathrm{~A} \\
(12,15)\end{array}$ & $\begin{array}{c}4,41 \\
(24,83)\end{array}$ & $\begin{array}{c}27,30 \mathrm{~A} \\
(8,69)\end{array}$ & $\begin{array}{c}0,16 \mathrm{~B} \\
(22,66)\end{array}$ & $\begin{array}{c}72,54 \mathrm{~A} \\
(3,33)\end{array}$ \\
\hline
\end{tabular}

Valores entre parênteses representam os coeficientes de variação (\%). Valores médios seguidos de mesma letra, nas colunas, não diferem entre si, pelo teste de Tukey, a 5\% de probabilidade.
Trugilho et al. (2005) encontraram teores maiores de cinza na madeira produzida mais externamente ao tronco das árvores, ou seja, na região do alburno. Esse fato pode explicar a diferença nos teores de cinza nas classes diamétricas, em que o maior teor ocorreu na menor classe, a que apresentou a maior porcentagem de alburno.

Vital et al. (1994), estudando a variabilidade genética das características de crescimento e das características da madeira e do carvão vegetal de 25 progênies de Eucalyptus camaldulensis Dehn, encontraram, em média, 32,36\% de carvão com 72,28\% de carbono fixo, $22,95 \%$ de material volátil e $0,77 \%$ de cinza.

Pelos dados da Tabela 2, verifica-se, ainda, que os teores de cinza e de carbono fixo apresentaram tendência de redução, enquanto o teor de materiais voláteis apresentou tendência de aumento com a classe diamétrica. Apesar da tendência de redução para o teor de carbono fixo e de aumento para o teor de materiais voláteis, com a classe diamétrica verifica-se que esse efeito não é significativo. Esse fato está de acordo com o normalmente observado na literatura (BOTREL et al., 2007), em que os teores de carbono e materiais voláteis são mais influenciados pela temperatura e pela taxa de aquecimento do sistema, o que, neste trabalho, foi constante para todos os tratamentos.

\subsection{Segunda carbonização - características quantitativas do carvão vegetal}

Os valores médios de rendimento gravimétrico para as amostras retiradas a 1,30 m do solo (DAP), em relação à posição radial, considerando os dois lados em relação à medula, para as três classes diamétricas em estudo, são apresentados na Tabela 3. Observa-se que os valores de rendimento gravimétrico tenderam a aumentar da casca para a medula, para todas as classes diamétricas. Nota-se, também, que os valores médios de rendimento gravimétrico em carvão vegetal tenderam a diminuir com a redução da classe diamétrica.

Considerando as três classes diamétricas, o valor médio de rendimento gravimétrico encontrado neste trabalho $(33,8 \%)$ foi próximo ao encontrado por Botrel et al. (2007), de 35,03\%, mas superior ao valor encontrado por Ribeiro e Machado (2005), estudando Eucalyptus urophylla, que foi de $26,91 \%$.

A classe diamétrica de $14,2 \mathrm{~cm}$ foi a que apresentou, de forma geral, os valores mais altos para o rendimento gravimétrico, com $34,2 \%$ e a classe diamétrica de $8,1 \mathrm{~cm}$ apresentou os menores valores médios, em torno de $33 \%$. Os valores de rendimento gravimétrico em carvão

Cerne, Lavras, v. 19, n. 3, p. 423-431, jul./set. 2013 
Tabela 3 - Valores médios de rendimento gravimétrico (\%) em carvão do clone de Eucalyptus grandis W. Hill ex Maiden x Eucalyptus urophylla $\mathrm{S}$. T. Blake, para as três classes diamétricas no DAP.

Table 3 - Average gravimetric yield (\%) values in charcoal of the Eucalyptus grandis W. Hill ex Maiden $x$ Eucalyptus urophylla S. T. Blake clone, for the three diametric classes at $\mathrm{DBH}$.

\begin{tabular}{|c|c|c|c|c|c|c|c|c|c|c|c|}
\hline \multirow{2}{*}{$\begin{array}{l}\text { Classe } \\
(\mathrm{cm})\end{array}$} & \multirow{2}{*}{$\begin{array}{c}\text { Lado } \\
\text { A }\end{array}$} & \multicolumn{9}{|c|}{ Distância da medula (cm) } & \multirow{2}{*}{$\begin{array}{c}\text { Lado } \\
\text { B }\end{array}$} \\
\hline & & 5,7 & 5,0 & 3,5 & 1,5 & 1,50 & 3,40 & 4,80 & 5,70 & 6,00 & \\
\hline \multirow[t]{3}{*}{14,2} & 34,20 & 32,31 & 32,97 & 35,40 & 36,13 & 36,40 & 35,88 & 33,08 & 33,06 & 32,39 & 34,16 \\
\hline & & \multicolumn{9}{|c|}{ Distância da medula (cm) } & \\
\hline & & & 3,9 & 2,8 & 1,1 & 1,3 & 2,9 & 4,0 & & & \\
\hline \multirow[t]{3}{*}{11,4} & 33,48 & & 32,10 & 33,09 & 35,23 & 35,68 & 34,83 & 32,58 & & & 34,36 \\
\hline & & \multicolumn{9}{|c|}{ Distância da medula (cm) } & \\
\hline & & & & 2,6 & 0,9 & 1,1 & 2,8 & & & & \\
\hline 8,1 & 33,11 & & & 32,82 & 33,40 & 33,13 & 32,92 & & & & 33,03 \\
\hline
\end{tabular}

vegetal encontrados neste trabalho estão de acordo com os relatados por Assis et al. (2008).

O gráfico da Figura 3 ilustra a variação radial média do rendimento gravimétrico para a classe 14,2 $\mathrm{cm}$. Observa-se que o comportamento da variação do rendimento gravimétrico é semelhante para os lados em relação à medula e que os maiores valores de rendimento gravimétricos ocorreram próximos á medula, havendo uma redução no sentido da casca. Trugilho et al. (2005) também verificaram uma tendência de maior produção de carvão vegetal nas posições intermediária e central das amostras carbonizadas.

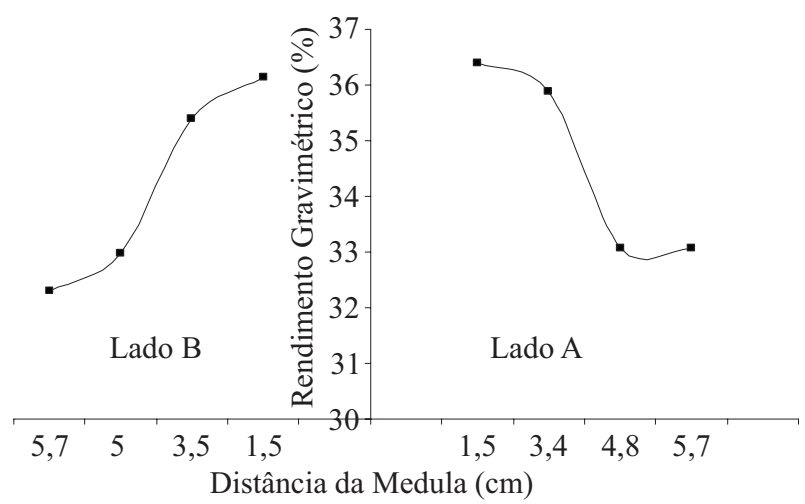

Figura 3 - Variação radial médio do rendimento gravimétrico de carvão do clone de Eucalyptus grandis W. Hill ex Maiden x Eucalyptus urophylla S. T. Blake, para a classe 14,2 cm.

Figure 3 - Average radial variation of the gravimetric yield of charcoal of the Eucalyptus grandis W. Hill ex Maiden $x$ Eucalyptus urophylla $S$. T. Blake clone, for the $14.2 \mathrm{~cm}$ diameter class.
$\mathrm{Na}$ Tabela 4, observam-se os valores médios de densidade relativa aparente do carvão para as amostras de madeira retiradas a $1,30 \mathrm{~m}$ do solo em relação à posição radial, considerando os dois lados em relação à medula, para as três classes diamétricas.

Observa-se que os valores médios de densidade relativa aparente aumentaram no sentido medula-casca, em todas as classes diamétricas. Observa-se também que não ocorreu diferença significativa entre os valores de densidade relativa aparente entre as classes diamétricas, com valor médio geral de $0,395 \mathrm{~g} / \mathrm{cm}^{3}$. A classe diamétrica de $11,4 \mathrm{~cm}$ apresentou valor médio de densidade relativa aparente ligeiramente inferior, de $0,390 \mathrm{~g} / \mathrm{cm}^{3}$.

Trugilho et al. (2005) também verificaram o mesmo comportamento de variação da densidade relativa aparente do carvão vegetal, ou seja, maior na posição radial externa e menor na posição radial central. Esse comportamento era esperado, uma vez que a densidade da madeira apresenta tendência de aumento do centro para a periferia do tronco.

Os gráficos da Figura 4 ilustram a variação radial média da densidade relativa aparente para a classe 14,2 cm (a). Observa-se similaridade entre os lados em relação à medula, o que também ocorre para a classe 11,4 cm (b), porém, com menor intensidade, o que pode ser explicado pelo número de amostras, as quais foram dependentes do diâmetro do disco.

Como se observa nos gráficos da Figura 4, os valores de densidade relativa aparente tendem a aumentar da medula para a casca, nas classes 14,2 e 11,4 cm, comportamento este inverso ao rendimento gravimétrico, que tende a diminuir com o afastamento da medula.

Cerne, Lavras, v. 19, n. 3, p. 423-431, jul./set. 2013 
Tabela 4 - Valores médios de densidade relativa aparente $\left(\mathrm{g} / \mathrm{cm}^{3}\right)$ do carvão do clone de Eucalyptus grandis W. Hill ex Maiden x Eucalyptus urophylla S. T. Blake, por classe diamétrica no DAP.

Table 4-Average apparent relative density $\left(\mathrm{g} / \mathrm{cm}^{3}\right)$ values of the charcoal of the Eucalyptus grandis W. Hill ex Maiden $x$ Eucalyptus urophylla S. T. Blake clone, for diametric class at DBH.

\begin{tabular}{|c|c|c|c|c|c|c|c|c|c|c|c|}
\hline \multirow{2}{*}{$\begin{array}{c}\text { Classe } \\
(\mathrm{cm})\end{array}$} & \multirow{2}{*}{$\begin{array}{c}\text { Lado } \\
\text { A }\end{array}$} & \multicolumn{9}{|c|}{ Distância da medula $(\mathrm{cm})$} & \multirow{2}{*}{$\begin{array}{c}\text { Lado } \\
\text { B }\end{array}$} \\
\hline & & 5,7 & 5 & 3,5 & 1,5 & 1,50 & 3,40 & 4,80 & 5,70 & 6,00 & \\
\hline \multirow[t]{3}{*}{14,2} & 0,400 & 0,488 & 0,444 & 0,341 & 0,327 & 0,341 & 0,336 & 0,454 & 0,454 & 0,325 & 0,396 \\
\hline & & \multicolumn{9}{|c|}{ Distância da medula (cm) } & \\
\hline & & & 3,9 & 2,8 & 1,1 & 1,3 & 2,9 & 4 & & & \\
\hline \multirow[t]{3}{*}{11,4} & 0,400 & & 0,471 & 0,378 & 0,351 & 0,326 & 0,376 & 0,435 & & & 0,379 \\
\hline & & \multicolumn{9}{|c|}{ Distância da medula (cm) } & \\
\hline & & & & 2,6 & 0,9 & 1,1 & 2,8 & & & & \\
\hline 8,1 & 0,394 & & & 0,430 & 0,358 & 0,349 & 0,453 & & & & 0,401 \\
\hline
\end{tabular}

(a)

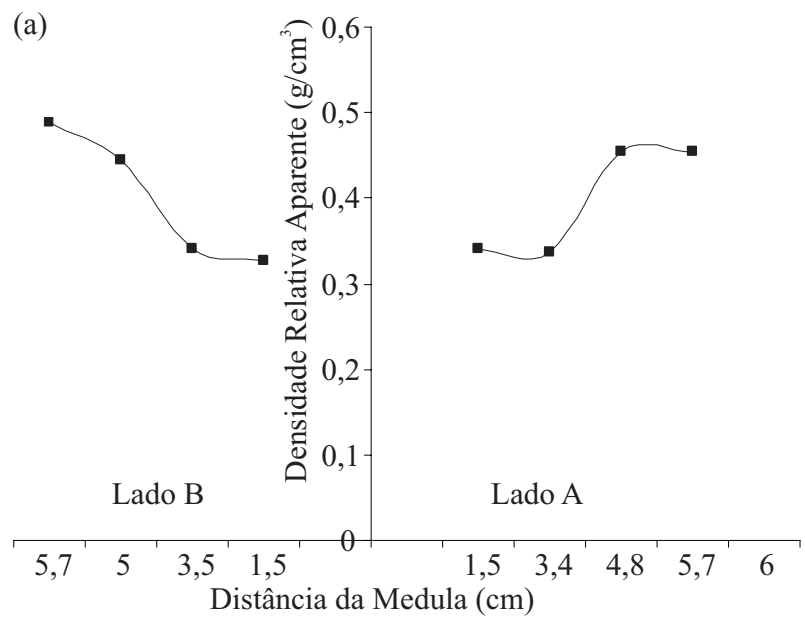

(b)

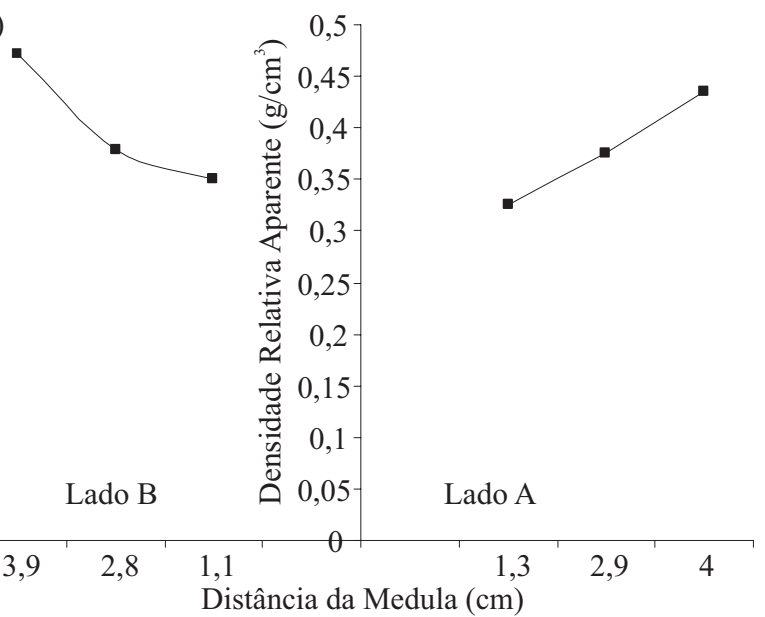

Figura 4 - Variação radial médio da densidade relativa aparente do carvão do clone de Eucalyptus grandis W. Hill ex Maiden x Eucalyptus urophylla S. T. Blake, para as classes 14,2 cm (a) e 11,4 cm (b).

Figure 4-Average radial variation of apparent relative density of the Eucalyptus grandis W. Hill ex Maiden $x$ Eucalyptus urophylla S. T. Blake clone charcoal, for the $14.2 \mathrm{~cm}$ (a) and 11,4 (b) classes.

Trugilho et al. (2005) também encontram valores mais elevados de densidade relativa aparente na região próxima à casca, sendo esse comportamento esperado, uma vez que a densidade da madeira apresenta tendência de aumento do centro para a periferia do tronco.

$\mathrm{Na}$ Tabela 5, são apresentados os valores médios de densidade relativa aparente do carvão no sentido radial e longitudinal. Verifica-se que os valores médios de densidade relativa aparente tendem a aumentar no sentido medula-casca e base-topo para as classes diamétricas, considerando os dois lados em relação à medula. Esse resultado foi menos percebido na classe diamétrica de
$11,4 \mathrm{~cm}$. Nota-se que, no sentido longitudinal, os valores médios de densidade relativa aparente para a classe $11,4 \mathrm{~cm}$ aumentaram até $10 \%$ da altura comercial, ocorrendo um decréscimo da densidade relativa aparente até 30\% e, a partir desse ponto, os valores tendem a aumentar até 70\% da altura comercial.

Como observado para os valores de densidade relativa aparente do carvão vegetal originado das amostras retiradas a 1,30 $\mathrm{m}$ do solo (DAP), a mesma variação da densidade relativa aparente ocorre para as amostras no sentido radial retiradas a $2 \%, 10 \%, 30 \%$ e $70 \%$ da altura comercial, para as três árvores em estudo, ou seja, maior

Cerne, Lavras, v. 19, n. 3, p. 423-431, jul./set. 2013 
Tabela 5 - Valores médios de densidade relativa aparente $\left(\mathrm{g} / \mathrm{cm}^{3}\right)$ do carvão no sentido radial e longitudinal.

Table 5 - Average apparent relative density $\left(\mathrm{g} / \mathrm{cm}^{3}\right)$ values of charcoal in the radial and longitudinal direction.

\begin{tabular}{|c|c|c|c|c|c|c|c|c|c|c|c|c|c|c|}
\hline \multirow{2}{*}{ Árvore } & \multirow{2}{*}{$\begin{array}{c}\text { Classe } \\
(\mathrm{cm})\end{array}$} & \multirow{2}{*}{$\begin{array}{c}\text { Altura } \\
(\%)\end{array}$} & \multirow{2}{*}{$\begin{array}{c}\text { Lado } \\
\text { A }\end{array}$} & \multicolumn{10}{|c|}{ Distância da medula (cm) } & \multirow{2}{*}{$\begin{array}{c}\text { Lado } \\
\text { B }\end{array}$} \\
\hline & & & & 6 & 5,7 & 5 & 3,5 & 1,5 & 1,5 & 3,4 & 4,8 & 5,7 & 6 & \\
\hline \multirow{7}{*}{1} & \multirow{5}{*}{14,2} & 2 & 0,411 & 0,477 & 0,458 & 0,428 & 0,357 & 0,335 & 0,309 & 0,312 & 0,366 & 0,434 & 0,461 & 0,377 \\
\hline & & 10 & 0,423 & 0,534 & 0,481 & 0,376 & 0,369 & 0,357 & 0,371 & 0,325 & 0,343 & 0,422 & 0,540 & 0,400 \\
\hline & & 30 & 0,426 & & 0,507 & 0,471 & 0,377 & 0,351 & 0,373 & 0,383 & 0,476 & 0,523 & & 0,439 \\
\hline & & 70 & 0,466 & & & 0,541 & 0,440 & 0,419 & 0,399 & 0,426 & 0,492 & & & 0,439 \\
\hline & & Média & & 0,505 & 0,482 & 0,454 & 0,386 & 0,366 & 0,363 & 0,361 & 0,420 & 0,460 & 0,501 & \\
\hline & & \multirow{2}{*}{$\begin{array}{c}\text { Altura } \\
(\%)\end{array}$} & \multirow{2}{*}{$\begin{array}{c}\text { Lado } \\
\text { A }\end{array}$} & \multicolumn{10}{|c|}{ Distância da medula (cm) } & Lado \\
\hline & & & & & 4,41 & 4,63 & 3,88 & 2,9 & 1,69 & 1,84 & 4,95 & 5,14 & & B \\
\hline \multirow{7}{*}{24} & \multirow{7}{*}{11,4} & 2 & 0,364 & & 0,458 & 0,412 & 0,347 & 0,237 & 0,365 & 0,369 & 0,403 & 0,472 & & 0,402 \\
\hline & & 10 & 0,414 & & 0,495 & 0,421 & 0,365 & 0,374 & 0,317 & 0,346 & 0,447 & 0,496 & & 0,402 \\
\hline & & 30 & 0,407 & & & 0,480 & 0,415 & 0,325 & 0,287 & 0,436 & 0,471 & & & 0,398 \\
\hline & & 70 & 0,404 & & & & 0,458 & 0,350 & 0,376 & 0,454 & & & & 0,415 \\
\hline & & Média & & & 0,476 & 0,438 & 0,397 & 0,322 & 0,336 & 0,401 & 0,440 & 0,484 & & \\
\hline & & \multirow{2}{*}{$\begin{array}{c}\text { Altura } \\
(\%)\end{array}$} & \multirow{2}{*}{$\begin{array}{c}\text { Lado } \\
\text { A }\end{array}$} & \multicolumn{10}{|c|}{ Distância da medula (cm) } & Lado \\
\hline & & & & & & 6,28 & 4,33 & 2,9 & 2,61 & 2,7 & 6,34 & & & B \\
\hline \multirow{5}{*}{48} & \multirow{5}{*}{8,1} & 2 & 0,371 & & & 0,440 & 0,367 & 0,305 & 0,313 & 0,368 & 0,420 & & & 0,367 \\
\hline & & 10 & 0,393 & & & 0,488 & 0,351 & 0,341 & 0,295 & 0,361 & 0,459 & & & 0,372 \\
\hline & & 30 & 0,406 & & & 0,485 & 0,374 & 0,361 & 0,342 & 0,370 & 0,483 & & & 0,398 \\
\hline & & 70 & 0,439 & & & & 0,510 & 0,367 & 0,545 & 0,506 & & & & 0,526 \\
\hline & & Média & & & & 0,471 & 0,400 & 0,344 & 0,374 & 0,401 & 0,454 & & & \\
\hline
\end{tabular}

na posição radial externa e menor na posição radial central. Esse comportamento já era esperado, uma vez que a densidade da madeira apresenta tendência de aumento do centro para a periferia do tronco, como descrito por Silva et al. (2004), Tomazello Filho (1987) e Trugilho et al. (2005).

Oliveira et al. (2005) estudaram a madeira de $C$. citriodora, E. cloeziana, E. paniculata, E. tereticornis, E. pilularis e E. urophylla e constataram que ocorreu queda da densidade no topo das árvores, o que não foi verificado neste trabalho, pois a amostragem ocorreu até $70 \%$ da altura comercial.

Alzate et al. (2005) também encontraram esse mesmo padrão de variação para madeira de E. grandis $x$ E. urophylla, ou seja, ocorreu um aumento da densidade básica no sentido base-topo.

O comportamento médio geral da densidade relativa aparente por posição longitudinal e classe diamétrica para as árvores em estudo pode ser mais bem visualizado no gráfico da Figura 5. Os maiores valores

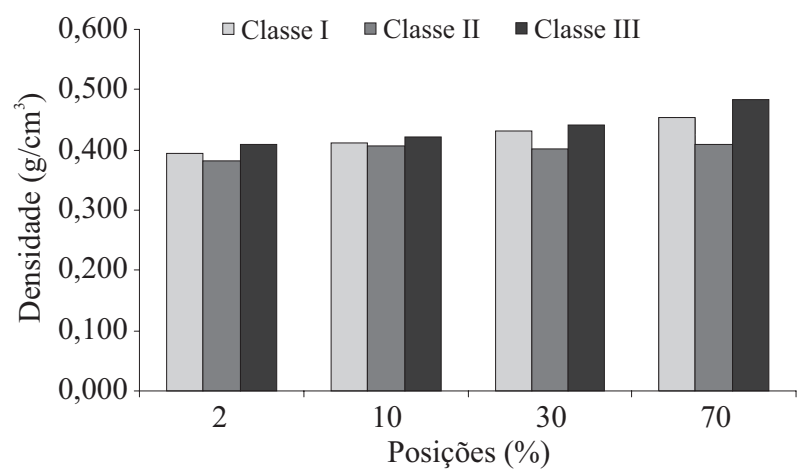

Figura 5 - Variação dos valores de densidade relativa aparente do carvão do clone de Eucalyptus grandis W. Hill ex Maiden x Eucalyptus urophylla S. T. Blake, por posição longitudinal e classe diamétrica.

Figure 5 - Variation of the apparent relative density values of the charcoal of the Eucalyptus grandis W. Hill ex Maiden $x$ Eucalyptus urophylla S. T. Blake clone, by longitudinal position and diametric class.

Cerne, Lavras, v. 19, n. 3, p. 423-431, jul./set. 2013 
médios de densidade relativa aparente do carvão ocorreram na árvore 1, pertencente à classe diamétrica de $14,2 \mathrm{~cm}$, com $0,423 \mathrm{~g} / \mathrm{cm}^{3}$, seguida pela árvore 48 , da classe $8,1 \mathrm{~cm}$, com $0,409 \mathrm{~g} / \mathrm{cm}^{3} \mathrm{e}$, por fim, a árvore 24 , pertencente à classe diamétrica de $11,4 \mathrm{~cm}$, com $0,401 \mathrm{~g} / \mathrm{cm}^{3}$.

Esse resultado está em conformidade com o encontrado por Pádua (2009). Esse autor, estudando a variação da densidade básica na madeira de um clone de híbrido de Eucalyptus grandis x Eucalyptus urophylla, encontrou a mesma tendência, ou seja, aumento da densidade básica no sentido medula-casca.

\section{CONCLUSÕES}

Primeira carbonização:

- a classe diamétrica influenciou, significativamente, o rendimento gravimétrico em carvão vegetal, em líquido pirolenhoso e o teor de cinza do carvão vegetal produzido;

- o rendimento em gás não condensável, o teor de materiais voláteis e o de carbono fixo não foram influenciados pela classe diamétrica;

- os teores de cinza e de carbono fixo apresentaram tendência de redução, enquanto o teor de materiais voláteis apresentou tendência de aumento em relação à classe diamétrica.

Segunda carbonização:

- o rendimento gravimétrico aumentou da casca para a medula, para todas as classes diamétricas e ocorreu redução dessa característica com a diminuição das classes diamétricas;

- os valores de densidade relativa aparente tenderam a aumentar no sentido medula-casca, em todas as classes diamétricas.

\section{REFERÊNCIAS}

ALZATE, S. B. A.; TOMAZELLO FILHO, M.; PIEDADE, S. M. de S. Variação longitudinal da densidade básica da madeira de clones de Eucalyptus grandis Hill ex Maiden, E. saligna Sm. e E. grandis x urophylla. Scientia Forestalis, Piracicaba, n. 68 , p. $87-95$, ago. 2005 .

ASSIS, C. O. de; TRUGILHO, P. F.; MENDES, L. M.; SILVA, J. R. M. da; LIMA, J. T. Sistema alternativo para carbonização de madeira. Scientia Forestalis, Piracicaba, v. 36, n. 78, p. 133-140, jun. 2008.

ASSOCIAÇÃO BRASILEIRA DE NORMAS TÉCNICAS. NBR 11941. Rio de Janeiro, 2003. 6 p.

Cerne, Lavras, v. 19, n. 3, p. 423-431, jul./set. 2013
ASSOCIAÇÃO BRASILEIRA DE NORMAS TÉCNICAS.

NBR 8112: carvão vegetal: análise imediata. Rio de Janeiro, $1983.5 \mathrm{p}$.

BOTREL, M. C. G.; TRUGILHO, P. F.; ROSADO, S. C. da S.; SILVA, J. R. M. da. Melhoramento genético das propriedades do carvão vegetal de Eucalyptus. Revista Árvore, Viçosa, v. 31, n. 3, p. 391-398, maio/jun. 2007.

EUCALIPTO na indústria de carvão vegetal. Revista da Madeira, Itajaí, n. 75, ago. 2003. Disponível em: <http://www. remade.com.br/br/revistadamadeira_materia.php?num $=395 \&$ subject $=$ Carvão $\% 20$ Vegetal\&title $=-\mathrm{O} \% 20$ eucalipto $\% 20$ na $\% 20$ indústria\%20de\%20carvão\%20vegetal $>$. Acesso em: 5 mar. 2008.

GOLDSTEIN, I. S. Wood technology: chemical aspects. Washington: America Chemical Society, 1977. 372 p.

MENDES, M. G.; GOMES, P. A.; OLIVEIRA, J. B. Propriedades e controle de qualidade do carvão vegetal. In: PENEDO, W. R. (Ed.). Produção e utilização do carvão vegetal. Belo Horizonte: CETEC, 1982. p. 76-89.

OLIVEIRA, J. T. da S.; SOUZA, L. C.; DELLA LÚCIA, R. M. Influência dos extrativos na resistência ao apodrecimento de seis espécies de madeira. Revista Árvore, Viçosa, v. 29, n. 5, p. 819-826, set./out. 2005.

PÁDUA, F. A. de. Amostragem para avaliação da densidade básica da madeira de um híbrido de Eucalyptus grandis W. Hill ex Maiden x Eucalyptus urophylla S. T.

Blake. 2009. 87 p. Tese (Doutorado em Ciência e Tecnologia da Madeira) - Universidade Federal de Lavras, Lavras, 2009.

PINHEIRO, P. C. da C.; FIGUEIREDO, F. J.; SEYE, O. Influência da temperatura e da taxa de aquecimento da carbonização nas propriedades do carvão vegetal de Eucalyptus. Biomassa \& Energia, Botucatu, v. 2, n. 2, p. 159168, jul./dez. 2005.

RIBEIRO, A. S.; MACHADO, A. de A. Carbonização de resíduos do processamento mecânico da madeira de eucalipto. Ciência Florestal, Santa Maria, v. 15, n. 1, p. 1-7, jan./fev. 2005.

SILVA, J. de C.; OLIVEIRA, T. da S.; TOMAZELLO FILHO, M.; KEINERT JÚNIOR, S.; MATOS, J. L. M. de. Influência da idade e da posição radial na massa específica da madeira de Eucalyptus grandis Hill ex. maiden. Revista Floresta, Curitiba, v. 34, n. 1, p. 13-22, jan./abr. 2004. 
TOMAZELLO FILHO, M. Variação radial da densidade básica em estrutura anatômica da madeira do Eucalyptus globulus, E. pellita, E. acmenioides. Instituto de Pesquisas e Estudos Florestais, Piracicaba, n. 36, p. 35-42, ago. 1987.

TRUGILHO, P. F.; SILVA, J. R. M.; LIMA, J. T.; MENDES, L. M.; MENDES, L. F. B. Rendimentos e características do carvão vegetal em função da posição radial da amostragem em clones de Eucalyptus. Cerne, Lavras, v. 11, n. 2, p. 178-186, jan./jun. 2005.

VITAL, B. R.; ALMEIDA, J. de; VALENTE, O. F.; PIRES, I. E. Características de crescimento das árvores e de qualidade da madeira de Eucalyptus camaldulensis para a produção de carvão. Instituto de Pesquisas e Estudos Florestais, Piracicaba, n. 47, p. 22-28, maio 1994.

Recebido: 19 de abril de 2011; aceito: 25 de janeiro de 2013.

Cerne, Lavras, v. 19, n. 3, p. 423-431, jul./set. 2013 
